\title{
Identification method for flexure and shear behavior of cantilever structures
}

\author{
F. Garcés ${ }^{\mathrm{a}, *}$, C. Genatios ${ }^{\mathrm{a}}$, P. García ${ }^{\mathrm{a}}$, A. Mebarki ${ }^{\mathrm{b}}$ and M. Lafuente ${ }^{\mathrm{a}}$ \\ ${ }^{a} I M M E$, University Central of Venezuela. IMME-UCV, apto postal 50.361, Caracas 1050-A, Venezuela \\ ${ }^{\mathrm{b}}$ Laboratoire de Mécanique, University of Marné la Vallée, Laboratoire de Mécanique, Université de Marne la \\ Vallée (France), 5 Bd Descartes, Cité Descartes, 77454 Marne la-Vallée, Cedex 2, France
}

Received 16 July 2007

Revised 6 June 2008

\begin{abstract}
This article presents an original identification method for the assessment of flexure and shear stiffness of cantilever structures or shear wall buildings. Required data include an initial (theoretical) model, an estimation of lumped mass values (by floor) and an experimental evaluation of two eigenvalues (modal frequencies and their modal shapes). The method estimates coefficients whenever flexural (EI) or shear (GA) values are relevant or irrelevant. An initial formula includes both shear and flexural components. Furthermore, particular developments are carried out for particular cases of irrelevant shear or flexural deformations. A numerical simulation of a real chimney is performed to study the effectiveness of the methodology in identifying damage under noise conditions. A dynamic-test experiment is carried out on a steel cantilever which suffers damage in two sections. The result obtained from the application of the proposed methodology is satisfactory in both numerical and experimental cases, identifying precisely the stiffness changes in the system.
\end{abstract}

Keywords: Modal analysis, damage identification, flexure behavior, stiffness reduction, cantilever structures

\section{Introduction}

There is always a difference between the theoretical model of a building and the real one. Structural stiffness can vary due to time degradation, building modifications, damage, overloads or seismic effects. Structural damage is not always visible, due to the level of damage or the difficulty of accessing the elements of the structure. For this reason, the vibration-based methods are promising because they are nondestructive and the vibration signal of a structure is easily measurable using properly installed sensors.

For damage-identification Rytter [1] methodology defines four levels of damage:

- Level 1: Determination if there is damage in the structure.

- Level 2: Level 1 plus determination of the geometric location of the damage.

- Level 3: Level 2 plus quantification of the severity of the damage.

- Level 4: Level 3 plus prediction of the remaining service life of the structure.

The existence of damage produces changes in the dynamic parameters of the system - vibrating frequencies, mode shapes, critical damping percentage, or its strain energy. The evaluation of the structure dynamics and its properties allows the determination the existence of any changes in any of its parameters which will signal whether there is damage in the structure or not [2-4]. The implementation of these studies also involves the evaluation of the vibration-modal frequencies, the study of the modal form curvature-changes [5], and the establishment of the correlation indexes (MAC [6], COMAC [7]) from these modal forms [8].

\footnotetext{
*Corresponding author. E-mail: francisco.garces@ucv.ve.
} 
Other methodologies for structural parameter estimation use the frequencies and vibration modes from measurements of undamaged condition and for the presence of damage by establishing changes in the mass and stiffness of the systems $[9,10]$. Other methods use matrix optimization instead of parametric coefficients to determine the stiffness matrix using the frequencies and vibration modes from measurements [9-12]. Similarly, there are some algorithms that, while using the optimization method to establish the structural damage, incorporate the Markov parameters [13].

Another method to determine changes in the stiffness of a structure is through probabilities, which is calculated by using the probability density function (pdf) of the system modal properties [14]. Other damage-detection methods are based on a previous structure modelling using finite and modal information obtained from the trial made to estimate the structural changes in each section of the system [9,15-17]. In general these methods are applied to any type of structure allowing the detection of a local damage in the structure; however, it is necessary to have various modal forms which should include all the coordinates in the finite-elements prototype. Due to this problem, the acquisition of reliable results implies that it is necessary to measure the frequencies and rotational vibration modes, which in some cases could be difficult.

There are algorithms that allow the location and quantification of the damage in certain structural cantilever typologies, shear buildings, and others [18-20]. By knowing the stiffness or flexibility matrix topology, we can reduce the number of modal forms and the necessary coordinates for the structural estimation. In general, this reduces the number of measurement points on the structures. This type of method adopts the methodology developed in this paper. The aim of this paper is to present and to demonstrate the efficacy of an identification procedure for flexural stiffness $(\mathrm{EI})$ and shear stiffness evaluation $(\mathrm{GA} / \gamma)$ for cantilever structures with predominant behavior on flexure, shear or both with the use of two modal forms and their frequencies. The methodology uses the knowledge of the matrices forms of a typical cantilever to simplify the identification method.

The shown algorithm has the advantage that it only requires the knowledge of two mode shapes with their respective frequencies (without doing the measurement of rotational coordinates, which generally are difficult to obtain). This implies that the proposed methodology is a powerful tool for the prompt decision about the future of this type of structure. This methodology has very wide usage with little amount of experimental measurement which drastically reduces the excessive costs and number of tests. The following paper presents the mathematical development of the identification methodology. This method is later applied to locate the stiffness changes in a numerically simulated chimney and the stiffness changes in a steel cantilever, which was previously studied in a free-vibration essay. Results illustrate that the proposed methodology successfully determines the location and the type of damage in the structure with accuracy.

\section{Shear and flexural stiffness evaluation for cantilever structures}

\subsection{General Methodology for flexural and shear stiffness evaluation}

Figure 1 shows the structural model considering flexural and shear behavior, rigid slabs, non vertical deformations and consistent masses. The structure is idealized with $N$ dynamic degrees of freedom (dof), with an unknown flexibility matrix $F$ (or its corresponding stiffness matrix $K$ ) and a known mass matrix $M$. Is accepted that the dynamic analysis allows the research of m modal frequencies and its corresponding mode shapes with $m<N$.

Consider the equation of motion for an undamped $\mathrm{n}$ degrees-of-freedom (dof) structure, given by:

$$
M \ddot{u}+K u=f(t)
$$

Where $M=$ mass matrix, $K=$ Stiffness Matrix, $\ddot{u}=$ acceleration vector, $u=$ displacement vector, $f(t)=$ excitation vector force. Consider the corresponding characteristics equation:

$$
\left(K-\lambda_{i} M\right) \phi_{i}=0
$$

The last equation can be rewritten as:

$$
\left(\lambda_{i}^{-1}-F \cdot M\right) \phi_{i}=0
$$



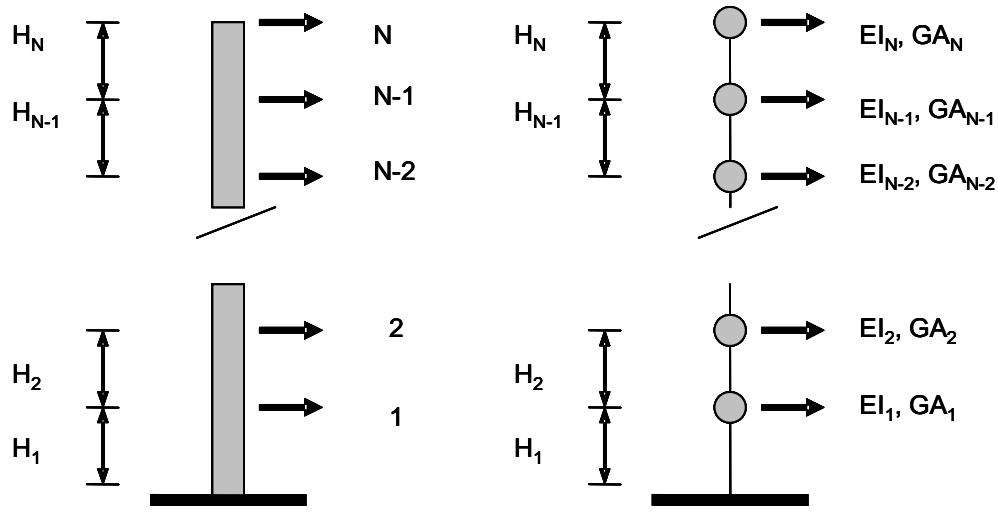

Fig. 1. $N$ dof structural model.

with $F=$ Flexibility matrix, $M=$ Mass matrix, $\lambda_{i}=\omega_{i}^{2}, \omega_{i}=i^{t h}$ modal frequency, $i=1$ to $N, \phi_{i}=i^{t h}$ eigenvector.

Equation (3) corresponds to:

$$
\left\{\left[\begin{array}{ccc}
1 / \lambda_{a} & \cdots & 0 \\
\vdots & 1 / \lambda_{a} & \vdots \\
0 & \cdots & 1 / \lambda_{a}
\end{array}\right]-\left[\begin{array}{ccc}
f_{11} & \cdots & f_{1 N} \\
\vdots & f_{i j} & \vdots \\
f_{N 1} & \cdots & f_{N N}
\end{array}\right]\left[\begin{array}{ccc}
m_{11} & \cdots & m_{1 N} \\
\vdots & m_{i j} & \vdots \\
m_{N 1} & \cdots & m_{N N}
\end{array}\right]\right\}\left(\begin{array}{c}
\phi_{a}^{1} \\
\vdots \\
\phi_{a}^{N}
\end{array}\right)=\left(\begin{array}{c}
0 \\
\vdots \\
0
\end{array}\right)
$$

with: $m_{i j}=i j$ coefficient of the matrix $M, f_{i j}=i j$ coefficient of the matrix $F, \phi_{a}^{i}=$ coordinate of the $i^{t h}$ level of mode shape a, $1 / \lambda_{a}=1 / \omega_{a}^{2}$ and $\omega_{a}$ modal frequency of mode " $a$ ".

Considering two eigenvalues corresponding to the modal frequencies $\omega_{a}$ and $\omega_{b}$, and the mode shapes $\phi_{a}$ and $\phi_{b}$, Eq. (2) becomes:

$$
\begin{gathered}
\left(f_{11} m_{11}+\ldots+f_{1 N} m_{N 1}\right) \phi_{a}^{1}+\ldots+\left(f_{11} m_{1 N}+\ldots+f_{1 N} m_{N N}\right) \phi_{a}^{N}=\phi_{a}^{1} / \omega_{a}^{2} \\
\left(f_{11} m_{11}+\ldots+f_{1 N} m_{N 1}\right) \phi_{b}^{1}+\ldots+\left(f_{11} m_{1 N}+\ldots+f_{1 N} m_{N N}\right) \phi_{b}^{N}=\phi_{b}^{1} / \omega_{b}^{2} \\
\vdots \\
\left(f_{N 1} m_{11}+\ldots+f_{N N} m_{N 1}\right) \phi_{a}^{1}+\ldots+\left(f_{N 1} m_{1 N}+\ldots+f_{N N} m_{N N}\right) \phi_{a}^{N}=\phi_{a}^{N} / \omega_{a}^{2} \\
\left(f_{N 1} m_{11}+\ldots+f_{N N} m_{N 1}\right) \phi_{b}^{1}+\ldots+\left(f_{N 1} m_{1 N}+\ldots+f_{N N} m_{N N}\right) \phi_{b}^{N}=\phi_{b}^{N} / \omega_{b}^{2}
\end{gathered}
$$

Each flexibility value can be evaluated as follows:

$$
f_{i j}=\sum_{k=1}^{N} \frac{1}{(E I)_{k}} \alpha_{i j k} \cdot 1_{k \leqslant M i n(i, j)}+\sum_{k=1}^{N} \frac{1}{\left(\frac{G A}{\gamma}\right)_{k}} \beta_{k} \cdot 1_{k \leqslant \operatorname{Min}(i, j)} \forall i, j
$$

with : $1_{k \leqslant \operatorname{Min}(i, j)}\left\{\begin{array}{c}1: \text { if } k \leqslant \operatorname{Min}(i, j) \\ 0: \text { otherwise }\end{array}\right.$

$$
\alpha_{i j k}=H_{k}\left[\left(\sum_{l=k+1}^{i} H_{l}\right)\left(\sum_{l=k+1}^{j} H_{l}\right)+\frac{H_{k}}{2}\left(\sum_{l=k+1}^{i} H_{l}+\sum_{l=k+1}^{j} H_{l}\right)+\frac{H_{k}^{2}}{3}\right] \quad \beta_{k}=H_{k}
$$

Where: $H_{k}=$ story height of level " $k$ ", $E_{k}=$ elasticity modulus of the material of level " $k$ ", $I_{k}=$ Inertia modulus of level " $k$ ", $G_{k}=$ shear modulus the material of level " $k$ ", $\left(A_{k} / \gamma\right)=$ shear transversal surface of level " $k$ ".

The goal of this procedure is the evaluation of the coefficients $(\mathrm{EI})_{k}$ and $(\mathrm{GA} / \gamma)_{k}$, for each level " $k$ ", with $k=1$ to $N$. Considering Eqs (5), (6) and (7) we come to:

$$
\left[\begin{array}{ccccc}
a_{11}^{a} & b_{11}^{a} & 0 & \cdots & 0 \\
a_{11}^{b} & b_{11}^{b} & 0 & \cdots & 0 \\
\vdots & \vdots & & \ddots & \vdots \\
a_{N, 1}^{a} & b_{N, 1}^{a} \cdots & a_{N N}^{a} & b_{N N}^{a} \\
a_{N, 1}^{b} & b_{N, 1}^{b} & \cdots & a_{N N}^{b} & b_{N N}^{b}
\end{array}\right]\left\{\begin{array}{c}
1 /(E I)_{1} \\
1 /(G A / \gamma)_{1} \\
\vdots \\
1 /(E I)_{N} \\
1 /(G A / \gamma)_{N}
\end{array}\right\}=\left(\begin{array}{c}
\phi_{a}^{1} / \omega_{a}^{2} \\
\phi_{b}^{1} / \omega_{b}^{2} \\
\vdots \\
\phi_{a}^{N} / \omega_{a}^{2} \\
\phi_{b}^{N} / \omega_{b}^{2}
\end{array}\right)
$$


The coefficients involved in Eq. (8) are:

$$
\begin{aligned}
a_{i k}^{a} & =\sum_{l=k}^{N} \sum_{u=1}^{N} m_{l u} \cdot \phi_{a}^{u} \cdot \alpha_{i j k} \cdot 1_{k \leqslant \operatorname{Min}(\mathrm{i}, \mathrm{j})} b_{i k}^{a}=\beta_{k} \cdot \sum_{l=k}^{N} \sum_{u=1}^{N} m_{l u} \cdot \phi_{a}^{u} 1_{k \leqslant \operatorname{Min}(i, j)} \quad \text { for } k=1 \text { to } N \\
a_{i k}^{b} & =\sum_{l=k}^{N} \sum_{u=1}^{N} m_{l u} \cdot \phi_{b}^{u} \cdot \alpha_{i j k} \cdot 1_{k \leqslant \operatorname{Min}(i, j)} b_{i k}^{b}=\beta_{k} \cdot \sum_{l=k}^{N} \sum_{u=1}^{N} m_{l u} \cdot \phi_{b}^{u} 1_{k \leqslant \operatorname{Min}(i, j)}
\end{aligned}
$$

Equation (8) defines a system of $2 \mathrm{~N}$ equations with $2 \mathrm{~N}$ unknowns, as two unknown coefficients (EI) and (GA/ $\gamma$ ) are considered for each level. This fact imposes the requirement of two mode shapes and their corresponding frequencies in order to produce two sets of $\mathrm{N}$ equations, so $2 \mathrm{~N}$ equations.

\subsection{One story structure and some singularities}

A one store system leads to a singularity. Given matrix $[A]$, Eqs (5) to (7):

$$
\left[\begin{array}{ll}
a_{11}^{a} & b_{11}^{a} \\
a_{11}^{b} & b_{11}^{b}
\end{array}\right]\left\{\begin{array}{c}
1 /(E I)_{1} \\
1 /(G A / \gamma)_{1}
\end{array}\right\}=\left(\begin{array}{c}
\phi_{a}^{1} / \omega_{a}^{2} \\
\phi_{b}^{1} / \omega_{b}^{2}
\end{array}\right)
$$

As there is only 1 dof $\omega_{a}=\omega_{b}=\omega$. The determinant is equal to:

$$
\left[\begin{array}{ll}
a_{1_{1}}^{a} & b_{11}^{a} \\
a_{11}^{b} & b_{11}^{b}
\end{array}\right]=m_{1}^{2} \frac{H_{1}^{4}}{3}\left(\phi_{a}^{1} \phi_{b}^{1}-\phi_{b}^{1} \phi_{1}^{1}\right)=0
$$

\subsection{General case and singularity in the highest level " $N$ ”}

The previous singularity is always found at the highest level " $N$ ", so the last two equations of Eq. (8) shall have a particular treatment. The solution for the first $N-1$ levels is $(i=1, N-1)$ :

$$
\left[\begin{array}{ccccc}
a_{11}^{a} & b_{11}^{a} & 0 & \ldots & 0 \\
a_{11}^{b} & b_{11}^{b} & 0 & \ldots & 0 \\
\vdots & & & & \vdots \\
a_{N-1,1}^{a} & b_{N-1,1}^{a} & a_{N-1, N-1}^{a} & b_{N-1, N-1}^{a} \\
a_{N-1,1}^{b} & b_{N-1,1}^{b} & a_{N-1, N-1}^{b} & b_{N-1, N-1}^{b}
\end{array}\right]\left\{\begin{array}{c}
1 /(E I)_{1} \\
1 /(G A / \gamma)_{1} \\
\vdots \\
1 /(E I)_{N-1} \\
1 /(G A / \gamma)_{N-1}
\end{array}\right\}=\left(\begin{array}{c}
\phi_{a}^{1} / \omega_{a}^{2} \\
\phi_{b}^{1} / \omega_{b}^{2} \\
\vdots \\
\phi_{a}^{N-1} / \omega_{a}^{2} \\
\phi_{b}^{N-1} / \omega_{b}^{2}
\end{array}\right)
$$

\subsection{Damage and residual properties}

It is assumed that the damage affecting the $k$-th storey stiffness might be expressed through the following damage indicators:

$$
\left\{\begin{array}{l}
(E I)_{i}=\left(1-D_{i}^{f}\right) \cdot(E I)_{i}^{0} \\
(G A / \gamma)_{i}=\left(1-D_{i}^{v}\right) \cdot(G A / \gamma)_{i}^{0}
\end{array}\right.
$$

Where:

$D_{i}^{f}=$ damage indicator that affects the "flexural coefficient" for storey " $i$ ";

$D_{i}^{v}=$ damage indicator that affects the "shear coefficient";

$(E I)_{i}^{0}=$ its initial "flexural coefficient";

$(G A / \gamma)_{i}^{0}=$ its initial "shear coefficient".

Due to the singularity of the sub-matrix involving the $N$-th storey, it is assumed that the same level of damage affects both the flexural and shear coefficient:

$$
D_{N}^{f}=D_{N}^{V}=1-\frac{a_{N N}^{b} \frac{1}{(E I)_{0}^{N}}+b_{N N}^{b} \frac{1}{(G A / \gamma)_{0}^{N}}}{\frac{\phi_{b}^{N}}{\omega_{b}^{2}}-\left(\sum_{k=1}^{N-1} a_{N k}^{b} \frac{1}{(E I)_{k}}+b_{N k}^{b} \frac{1}{(G A / \gamma)_{k}}\right)}
$$

Once the residual factor values $(E I$ and $G A$ ) for each storey are known, the stiffness matrix is completely known. 


\subsection{Flexure coefficient evaluation}

Structures with flexure predominant behavior (so shear terms can be neglected), only (EI) terms are significant. In this case only one eigenpair is required so the flexure coefficient values can be obtained from the simplified system of equations obtained from Eq. (8):

$$
\left[\begin{array}{ccccc}
a_{11}^{a} & 0 & \cdots & \cdots & 0 \\
\vdots & \ddots & \ddots & \cdots & \vdots \\
a_{i 1}^{a} & \vdots & a_{i i}^{a} & \ddots & \vdots \\
\vdots & & \cdots & \ddots & 0 \\
a_{N, 1}^{a} & \cdots & a_{N i}^{a} & \cdots & a_{N N}^{a}
\end{array}\right]\left\{\begin{array}{c}
1 /(E I)_{1} \\
\vdots \\
1 /(E I)_{i} \\
\vdots \\
1 /(E I)_{N}
\end{array}\right\}=\left(\begin{array}{c}
\phi_{a}^{1} / \omega_{a}^{2} \\
\vdots \\
\phi_{a}^{i} / \omega_{a}^{2} \\
\vdots \\
\phi_{a}^{N} / \omega_{a}^{2}
\end{array}\right)
$$

\subsection{Shear coefficient evaluation}

In the case of significant values of shear coefficient compared to the flexure coefficient so this last can be neglected, only $(\mathrm{GA} / \gamma)$ terms are significant. Only one eigenpair is required and shear coefficient coefficients are given by:

$$
\left[\begin{array}{ccccc}
b_{11}^{a} & 0 & \cdots & \cdots & 0 \\
\vdots & \ddots & \ddots & \cdots & \vdots \\
b_{i 1}^{a} & \vdots & b_{i i}^{a} & \ddots & \vdots \\
\vdots & & \cdots & \ddots & 0 \\
b_{N, 1}^{a} & \cdots & b_{N i}^{a} & \cdots & b_{N N}^{a}
\end{array}\right]\left\{\begin{array}{c}
1 /(G A / \gamma)_{1} \\
\vdots \\
1 /(G A / \gamma)_{i} \\
\vdots \\
1 /(G A / \gamma)_{N}
\end{array}\right\}=\left(\begin{array}{c}
\phi_{a}^{1} / \omega_{a}^{2} \\
\vdots \\
\phi_{a}^{i} / \omega_{a}^{2} \\
\vdots \\
\phi_{a}^{N} / \omega_{a}^{2}
\end{array}\right)
$$

\section{Numerical study}

Before conducting experimental verification, first the effectiveness of the identification method was evaluated through numerical simulated damage identification. Simulated real steel chimney without damage and with assumed damage sections are considered. Figure 2 shows the geometric values of the steel chimney employed for the numerical study, from Ambrosini et al. [21]. The chimney is a cylindrical steel structure of $28 \mathrm{~m}$ high with $0.914 \mathrm{~m}$ diameter, cross section is $12 \mathrm{~mm}$ at the base and $3 \mathrm{~mm}$ at the top (Fig. 2). The simulated steel chimney is divided into 10 two-dimensional sections (Fig. 2). Three sections (No. 2, 5 and 8) are assumed to be subjected to $10 \%, 30 \%$ and $10 \%$ stiffness reduction. The modal data (mode shapes and frequencies) before and after damage (Fig. 3) have been calculated by using the finite element program SAP2000 [23].

\subsection{Effects of errors in dynamics measurements}

In order to study the effect of noise on the measurement of mode shapes and frequencies for the stiffness identification method, the mode shapes obtained from the numerical simulation were corrupted using the Sohn and Law algorithm [24]:

$$
\phi_{c}(n)=\phi\left(1+\frac{p}{100} R\right)
$$

Where $\phi_{c}(n)$ is the corrupted mode shape, $\phi$ is the uncorrupted mode shape obtained from numerical simulations, $p$ is a specified percentage of noise level, and $R$ is a random number between 0 and 1 . A set of ten vibration tests was carried out. Normally distributed random number from Matlab [25] was used.

Three study cases were proposed to study the effect of measurement noise on the damage identification:

Case a: frequency is corrupted and modal shape uncorrupted

Case b: frequency is uncorrupted and modal shape corrupted

Case c: frequency is corrupted and modal shape corrupted with same noise level.

Six values noise level were considered: $0.1 \%, 0.5,1,2 \%, 5 \%$ and $10 \%$. 

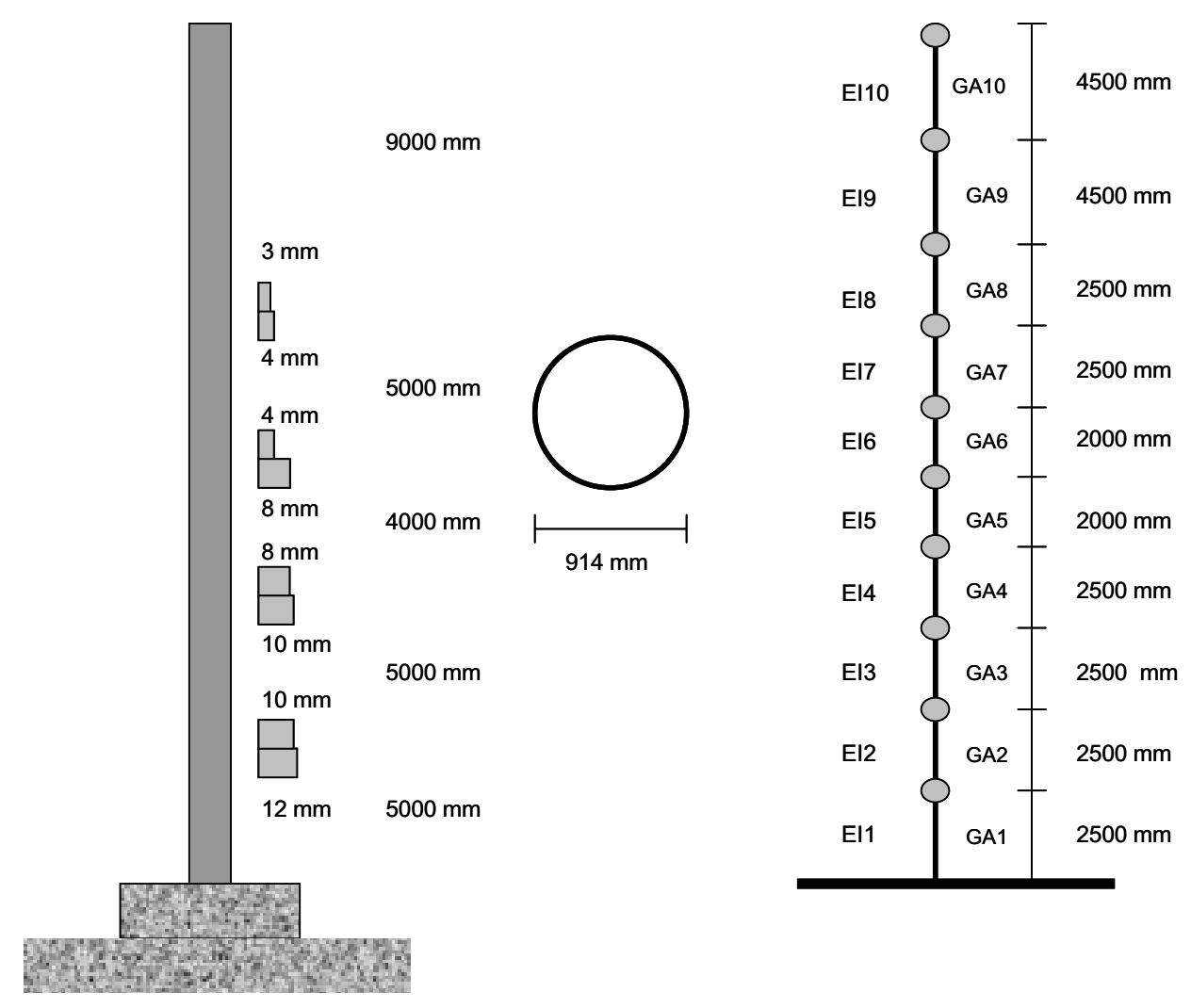

Fig. 2. A simulated steel chimney [21].
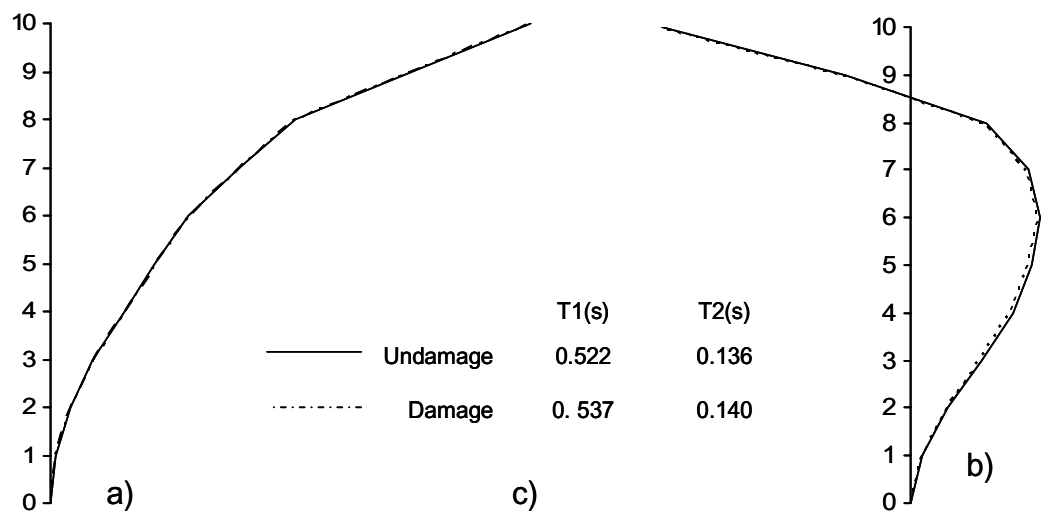

Frist mode shape

Second mode shape

Fig. 3. Modes and periods of the chimney.

\subsection{Results}

The stiffness identification is carried out using Eqs (12) and (14) with noisy mode shapes and mass matrix, the coefficients $E I$ and $G A$ were obtained for undamaged and damaged states.

The damage identification with different level of noise in frequency or mode shapes was performed to verify the efficiency and accuracy of the proposed method. The accuracy of the method is evaluated using the following expressions: 
Table 1

Relative error coefficients EI case a

\begin{tabular}{crrrrrr}
\hline \multirow{2}{*}{ Section } & \multicolumn{7}{c}{ Noise Level } \\
\cline { 2 - 7 } & $0.1 \%$ & $0.5 \%$ & \multicolumn{1}{c}{$1 \%$} & \multicolumn{1}{c}{$2 \%$} & \multicolumn{1}{c}{$5 \%$} & \multicolumn{1}{c}{$10 \%$} \\
\hline 1 & 0.00 & 0.00 & 0.00 & 0.00 & 0.00 & -0.01 \\
2 & 0.00 & 0.00 & 0.00 & 0.00 & 0.00 & -0.01 \\
3 & 0.00 & 0.00 & 0.00 & 0.00 & 0.00 & 0.00 \\
4 & 0.00 & 0.00 & 0.00 & 0.00 & 0.01 & -0.04 \\
5 & 0.00 & -0.01 & 0.00 & -0.01 & -0.01 & 0.13 \\
6 & 0.00 & 0.00 & 0.00 & 0.00 & 0.00 & 0.03 \\
7 & 0.00 & 0.00 & 0.00 & 0.00 & 0.01 & -0.06 \\
8 & 0.00 & 0.00 & 0.00 & 0.00 & -0.01 & 0.04 \\
9 & 0.00 & 0.00 & 0.00 & 0.00 & 0.00 & 0.01 \\
10 & 0.00 & 0.00 & 0.00 & 0.00 & 0.00 & 0.03 \\
\hline
\end{tabular}

Table 2

Relative error coefficients GA case a

\begin{tabular}{crrrrrr}
\multicolumn{7}{c}{ Relative error coefficients GA case a } \\
\hline Section & \multicolumn{7}{c}{ Noise Level } \\
\cline { 2 - 7 } & $0.1 \%$ & $0.5 \%$ & \multicolumn{1}{c}{$1 \%$} & \multicolumn{1}{c}{$2 \%$} & \multicolumn{1}{c}{$5 \%$} & $10 \%$ \\
\hline 1 & 0.00 & 0.00 & 0.00 & 0.00 & 0.01 & 0.00 \\
2 & 0.00 & -0.01 & 0.01 & 0.10 & 0.12 & 0.06 \\
3 & 0.00 & -0.02 & -0.01 & 0.12 & 0.17 & -0.15 \\
4 & 0.00 & -0.01 & 0.00 & -0.31 & -0.50 & 0.08 \\
5 & 0.04 & -0.40 & -0.56 & -2.47 & -3.43 & -4.13 \\
6 & 0.01 & -0.07 & -0.26 & -0.64 & -0.79 & -4.05 \\
7 & -0.01 & 0.07 & 0.12 & 0.13 & 0.32 & 0.85 \\
8 & -0.04 & 0.00 & 0.03 & -0.29 & 0.96 & 1.44 \\
9 & -0.10 & -0.01 & 0.37 & 0.06 & -0.38 & 3.15 \\
10 & 0.35 & -0.79 & -1.78 & -1.36 & 3.01 & 8.41 \\
\hline
\end{tabular}

Table 3

Relative error coefficients EI case b

\begin{tabular}{crrrrrr}
\hline Section & \multicolumn{7}{c}{ Noise Level } \\
\cline { 2 - 7 } & $0.1 \%$ & $0.5 \%$ & \multicolumn{1}{c}{$1 \%$} & \multicolumn{1}{c}{$2 \%$} & \multicolumn{1}{c}{$5 \%$} & \multicolumn{1}{c}{$0 \%$} \\
\hline 1 & 0.00 & 0.00 & 0.00 & 0.00 & 0.01 & 0.00 \\
2 & 0.00 & -0.01 & 0.01 & 0.10 & 0.12 & 0.06 \\
3 & 0.00 & -0.02 & -0.01 & 0.12 & 0.17 & -0.15 \\
4 & 0.00 & -0.01 & 0.00 & -0.31 & -0.50 & 0.08 \\
5 & 0.04 & -0.40 & -0.56 & -2.47 & -3.43 & -4.13 \\
6 & 0.01 & -0.07 & -0.26 & -0.64 & -0.79 & -4.05 \\
7 & -0.01 & 0.07 & 0.12 & 0.13 & 0.32 & 0.85 \\
8 & -0.04 & 0.00 & 0.03 & -0.29 & 0.96 & 1.44 \\
9 & -0.10 & -0.01 & 0.37 & 0.06 & -0.38 & 3.15 \\
10 & 0.35 & -0.79 & -1.78 & -1.36 & 3.01 & 8.41 \\
\hline
\end{tabular}

$$
\begin{aligned}
& \Delta E I=\frac{(E I)_{f}}{(E I)_{i}}, \quad \text { Relative Error }=\left(\frac{(\Delta E I)^{\text {estimated }}-(\Delta E I)^{\text {reference }}}{(\Delta E I)^{\text {reference }}}\right) \cdot 100 \\
& \Delta G A=\frac{(G A)_{f}}{(G A)_{i}}, \quad \text { Relative Error }=\left(\frac{(\Delta G A)^{\text {estimated }}-(\Delta G A)^{\text {reference }}}{(\Delta G A)^{\text {reference }}}\right) \cdot 100
\end{aligned}
$$

Where,

$E I_{i}^{\text {reference }}=$ flexure coefficient for level " $i$ " (FEM values);

$E I_{i}^{\text {estimated }}=$ the estimated flexure coefficient for level " $i$ ";

$G A_{i}^{\text {reference }}=$ shear coefficient for level " $i$ " (FEM values);

$G A_{i}^{\text {estimated }}=$ the estimated shear coefficient for level " $i$ ".

Tables 1 to 6 show the relative errors in the damage identification for the defined cases in Section 3.1. The following observations can be made: 
Table 4

Relative error coefficients GA case b

\begin{tabular}{crrrrrr}
\hline Section & \multicolumn{7}{c}{ Noise Level } \\
\cline { 2 - 7 } & $0.1 \%$ & $0.5 \%$ & \multicolumn{1}{c}{$1 \%$} & \multicolumn{1}{c}{$2 \%$} & \multicolumn{1}{c}{$5 \%$} & \multicolumn{1}{c}{$10 \%$} \\
\hline 1 & 0.00 & 0.01 & -0.05 & -0.12 & -0.28 & 0.01 \\
2 & -0.02 & 0.48 & -0.02 & -0.66 & -1.90 & 7.73 \\
3 & 0.00 & -0.20 & 0.17 & -0.38 & -1.44 & -0.80 \\
4 & -0.03 & -0.09 & -0.11 & -0.72 & 0.77 & -0.16 \\
5 & -0.93 & -3.01 & 4.20 & 5.10 & 3.03 & 1.72 \\
6 & -0.18 & -2.42 & -0.08 & -5.43 & 3.57 & 3.98 \\
7 & 0.39 & -0.75 & 1.48 & 3.52 & -0.79 & 3.99 \\
8 & 0.59 & -1.29 & -2.17 & -1.32 & 0.77 & -3.13 \\
9 & 1.23 & -4.60 & 5.49 & 3.13 & 3.73 & 6.52 \\
10 & 0.35 & -0.79 & -1.78 & -1.36 & 3.01 & 8.41 \\
\hline
\end{tabular}

Table 5

Relative error coefficients EI case c

\begin{tabular}{crrrrrr}
\hline Section & \multicolumn{7}{c}{ Noise Level } \\
\cline { 2 - 7 } & \multicolumn{1}{c}{$0.1 \%$} & \multicolumn{1}{c}{$0.5 \%$} & \multicolumn{1}{c}{$1 \%$} & \multicolumn{1}{c}{$2 \%$} & \multicolumn{1}{c}{$5 \%$} & \multicolumn{1}{c}{$10 \%$} \\
\hline 1 & 0.00 & 0.00 & 0.00 & 0.00 & 0.00 & 0.01 \\
2 & 0.00 & 0.02 & 0.01 & -0.06 & -0.27 & -0.02 \\
3 & 0.00 & 0.02 & 0.04 & -0.03 & -0.09 & 0.28 \\
4 & 0.00 & 0.02 & -0.11 & 0.17 & -0.19 & -0.80 \\
5 & 0.01 & -0.60 & -1.19 & 2.01 & -3.69 & -8.11 \\
6 & 0.02 & -0.08 & -0.38 & 0.25 & -2.12 & -2.34 \\
7 & -0.01 & 0.06 & 0.12 & -0.06 & 0.17 & 1.31 \\
8 & -0.02 & -0.01 & 0.09 & -0.07 & -0.28 & 1.22 \\
9 & 0.01 & -0.01 & -0.03 & -2.96 & 0.49 & 8.00 \\
10 & -0.09 & -0.10 & 0.76 & 4.31 & 0.76 & 5.75 \\
\hline
\end{tabular}

Table 6

Relative error coefficients GA case c

\begin{tabular}{crrrrrr}
\hline \multirow{2}{*}{ Section } & \multicolumn{7}{c}{ Noise Level } \\
\cline { 2 - 7 } & \multicolumn{1}{c}{$0.1 \%$} & \multicolumn{1}{c}{$0.5 \%$} & \multicolumn{1}{c}{$1 \%$} & \multicolumn{1}{c}{$2 \%$} & \multicolumn{1}{c}{$5 \%$} & \multicolumn{1}{c}{$10 \%$} \\
\hline 1 & 0.00 & -0.13 & 0.07 & -0.01 & 0.08 & -0.29 \\
2 & -0.02 & 1.11 & -0.93 & 0.16 & -1.74 & -3.03 \\
3 & 0.05 & -1.47 & 0.48 & -0.26 & 0.52 & -1.80 \\
4 & -0.05 & -3.19 & 1.11 & -2.73 & 1.84 & 1.49 \\
5 & 0.01 & 1.61 & -6.13 & 0.64 & -8.79 & -8.43 \\
6 & -0.37 & -3.17 & -0.82 & 0.85 & 5.79 & 3.96 \\
7 & 0.35 & -0.60 & 2.12 & -3.06 & 4.25 & 3.93 \\
8 & -0.40 & 5.78 & 0.21 & -0.32 & -1.87 & -7.14 \\
9 & -0.78 & 0.62 & 1.46 & 5.60 & 18.90 & 5.93 \\
10 & -0.09 & -0.10 & 0.76 & 4.31 & 0.76 & 5.75 \\
\hline
\end{tabular}

1. The quality of the damage identification is more sensitive to the perturbation of the mode shapes than that of frequencies.

2. The estimation of coefficients GA is made with less precision than coefficients EI. As expected a better adjustment of the flexural coefficient was obtained than the shear coefficient, for the chimney is mainly characterized by a flexural behavior. Numerical simulations for the shear wall buildings shows similar precision in the identification of flexural and shear coefficients [18].

3. The damage identification with level noise in the modal parameters give a reasonable agreement between the damage estimated and damage assumed. For the EI coefficients, in all cases the methodology identifies with precision the location of the stiffness changes as well as the variation of stiffness. For the study noise level the relative error of the estimation remain smaller that $9 \%$.

4. The Shear coefficients (GA) are identified with acceptable precision. Only the large error was present and was not corrected, for example in section 8 case $\mathrm{c}$ with noise level 5\%, the obtained error is closed to $20 \%$. 


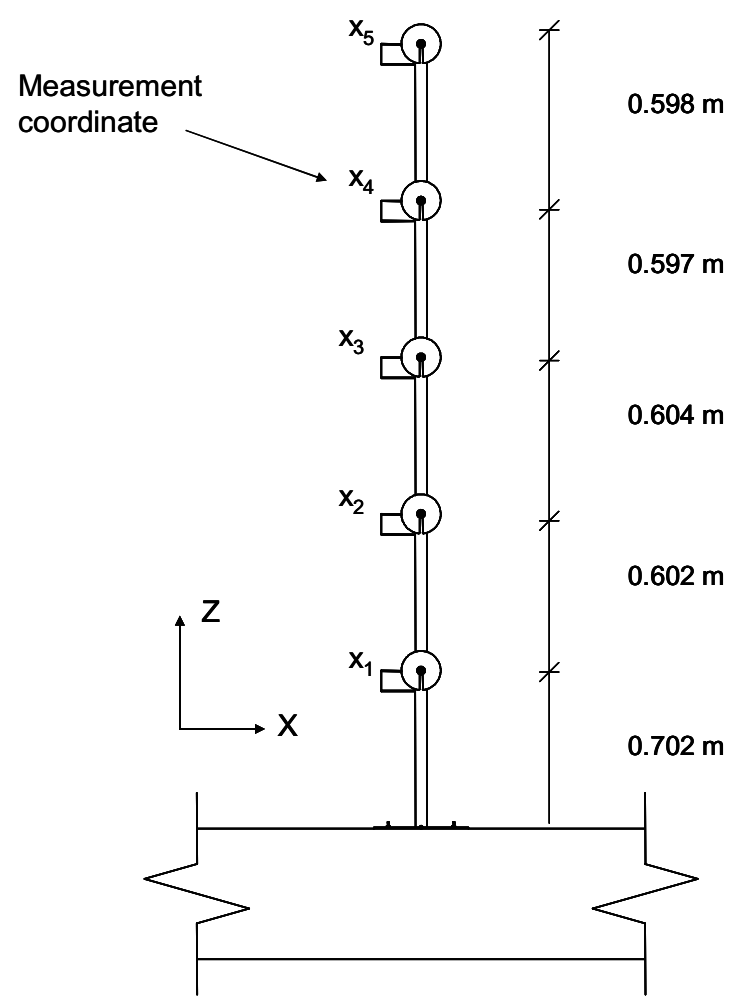

Fig. 4. Tested Model. $L=3.13 \mathrm{~m}, A=0.0757 \mathrm{~m}^{2}, I_{x x}=6.29 \times 10^{-4} \mathrm{~m}^{4}, E=205.4 \mathrm{GPa}, W$ (model and accelerometers) $=24.76 \mathrm{~kg}$.

\section{Experimental assessment}

\subsection{Model tested}

The model test item is a wide-flanged steel I-beam (IPN 80), consisting of a $80 \mathrm{~mm}$ deep web and a $42 \mathrm{~mm}$ wide flange. The Beam is 3.13 meters in length, divided in 5 sections. The model was fixed at the reinforced concrete beam (Fig. 4).

In order to obtain lower values of the modal frequencies and to obtain more mode shapes with the equipment available, the beam was excited in axis X direction (Fig. 4). Previous numerical simulations established a predominant flexural behavior for the beam. For this reason, the experimental study is limited only for estimate coefficients EI for each section.

\subsection{Experimental analysis and dynamic properties identification}

The beam was submitted to a free vibration test applying initial displacement or velocity conditions in each coordinates of measurement. As a result, a total of 25 responses in the lateral direction (along the $\mathrm{X}$-axis) were recorded in one series. Vibration responses were registered with unidirectional accelerometers Kinemetrics FBA-11 and processed with an Altus K2 Kinemetrics signal processing device. Measurements were taken for a frequency range of $0-50 \mathrm{~Hz}$. and the dynamic response was captured by 5 accelerometers, obtaining three of the five fundamentals frequencies of the model. Figure 5 presents a schematic representation of output-only modal identification. During dynamic testing, the measurement data was sampled at $f_{s}=250 \mathrm{~Hz}$, for 8192 data points for each channel. The dynamic properties were assessed using SADEX: a software to process structural dynamic signals in experimental tests [25]. 


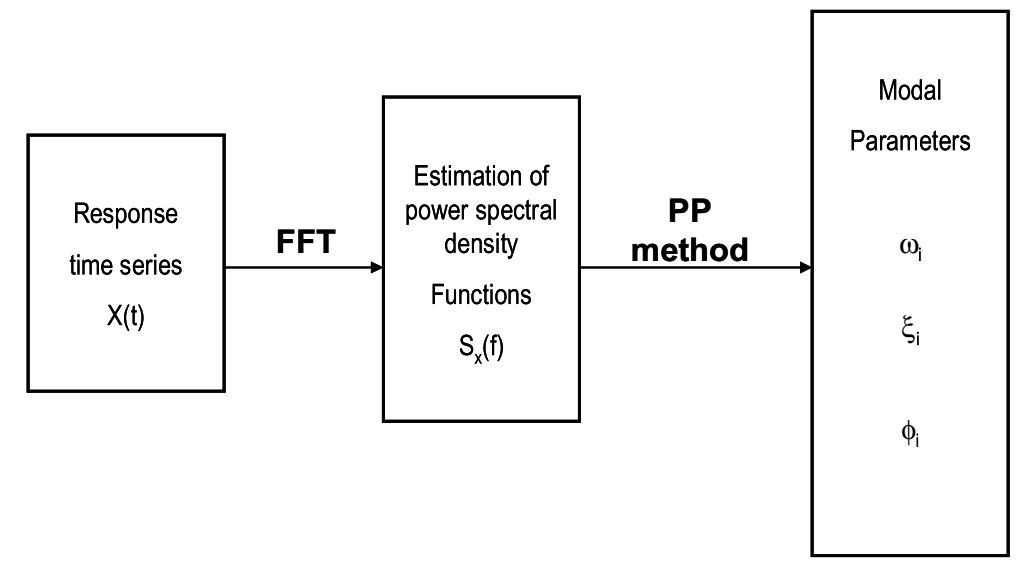

Fig. 5. Schematic representation of output-only modal identification.

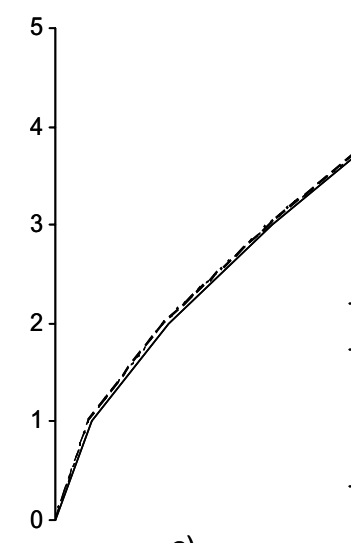

a)

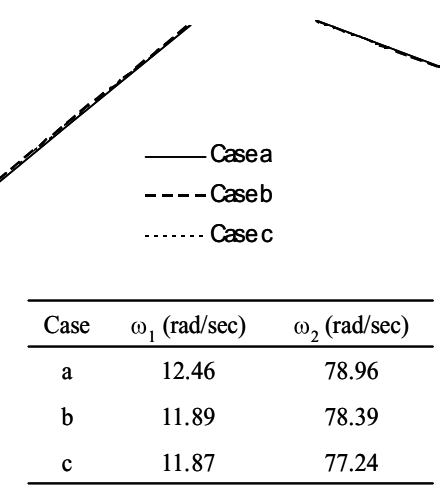

c)

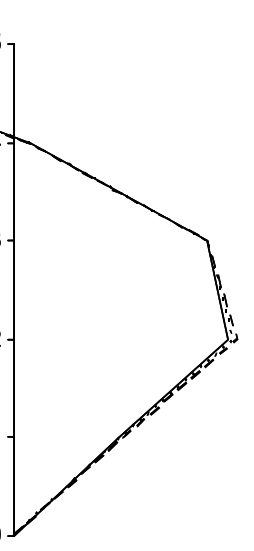

b)

Fig. 6. Modes and frequencies of free vibration of the cantilever.

\section{Study cases and estimation results}

Consecutive damage was introduced to the beam, in order to evaluate the proposed methodology. Saws were made in the beam flanges. These saws were localized in the half of the section. Three study cases were established: Case a: Initial undamaged beam (reference for evaluation of coefficient change), Case b: Beam with damage in the 2nd section and Case c: Beam with damage in the 2nd and 4th sections.

For each of the cases described above, free vibration tests were performed with excitation in different coordinates, in order to obtain records and to choose those with more quality and information. Dynamic properties were obtained for every case: Modal frequencies and mode shapes (Fig. 6). Once the beam mass is estimated and the experimental data analyzed, a linear system is formed (8). Solving the system (5) we obtain changes of coefficient for every case in relation to the initial structure (case a).

Table 7 shows the results of damage estimation in case b, and c. In each of the two studied cases, the method shows the location of the damage; in the case of undamaged sections, the maximum error estimation is $3 \%$, in relation to reference values (case a).

An important advantage of this approach is the need of only two mode shapes and simple dynamic tests. The references show that it is possible to extract two modal shapes by using ambient vibrations, free vibration and forced vibrations. The present method is affected by a high noise level in the measures [26]. An adequate signal processing will reduce significantly the noise influence in the modal shapes. 
Table 7

Coefficient changes estimation of the cantilever

\begin{tabular}{ccc}
\hline Section & $\begin{array}{c}\text { Case b } \\
E I_{f} / E I_{o}\end{array}$ & $\begin{array}{c}\text { Case c } \\
E I_{f} / E I_{o}\end{array}$ \\
\hline 1 & 1.00 & 1.00 \\
2 & 0.72 & 0.72 \\
3 & 1.01 & 1.01 \\
4 & 0.97 & 0.88 \\
5 & 1.02 & 1.00 \\
\hline
\end{tabular}

\section{Conclusion}

The damage identification procedure for cantilever structures was proposed. This methodology requires a known mass matrix and two natural frequencies with their corresponding mode shapes.

The stiffness identification procedure was illustrated with a numerical example of a real chimney, achieving good precision for stiffness changes in each section under different noise signal conditions. In addition, the stiffness estimation methodology was applied in an experimental study of a steel cantilever beam. Damage was performed in two sections of the beam. The method identified with precision the stiffness change as well as the damage location.

This approach can be applied in cantilever structures (chimneys, control towers, grandstands roofs, etc.). An important advantage is the need of only two mode shapes and simple dynamic tests. This method requires only unidirectional coordinates; does not require rotational coordinates and refined FE models. Therefore, it is convenient to apply this approach in order to identify structural cantilever typologies.

\section{References}

[1] A. Rytter, Vibration Based Inspection of Civil Engineering Structures, PhD Thesis, Aalborg University 1993.

[2] C. Genatios, G. Cascante, M. Lafuente, P. Bellan and M. Lorrain, Evaluation expérimentale des caractéristiques modales et ajustement du modèle dynamique d'un bâtiment de portiques, Rev Materials and Structures RILEM 32 (1999), 117-124.

[3] M. Breccolotti, G. Franceschini and A.L. Materazzi, Sensitivity of dynamic methods for damage detection in structural concrete bridges, Shock and Vibration 11(3-4) (2004), 383-394.

[4] A. Dimarogonas, Vibration of cracked structures: a state of the art review, Applied Mechanics Review 55 (1996), 831-857.

[5] A. Pandey, M. Biswas and M. Samman, Damage detection from changes in curvature mode shapes, Journal of Sound and Vibration 145 (1991), 321-332.

[6] W. D'Ambrogio and A. Fregolent, Dynamic model updating using virtual antiresonances, Shock and Vibration 11(3-4) (2004), 351-363.

[7] N. Lieven and D. Ewins, Spatial correlation of mode shapes coordinate modal assurance criterion (COMAC), Proc 6th Int Modal Analysis Conference 1 (1988), 690-695.

[8] R. Sampaio, M. Maia and M. Silva, Damage detection using the frequency-response-function curvature method, Journal of Sound and Vibration 226 (1999), 1029-1042.

[9] A. Yan and J.-C. Golinval, Structural damage localization by combining flexibility and stiffness methods, Engineering Structures 27 (2005), $1752-1761$.

[10] M. Baruch, Optimization Procedure to Correct Stiffness and Flexibility Matrices Using Vibrations Test, AIAA Journal 16 (1978), 12081210 .

[11] C. Genatios, F. Garcés, P. Bellan and M. Lafuente, Direct Matrix Adjustement in Experimental Dynamics, Proc. 11 European Conference on Earthquake Engineering, Paris, 1998.

[12] A.M. Kabe, Stiffness Matrix Adjustment Using Mode Data, AIAA Journal 23 (1985), 1431-1436.

[13] S. Hassiotis, Identification of damage using natural frequencies and Markov parameters, Computers and Structures 74 (2000), 365-373.

[14] L. Papadopoulos and E. García, Structural damage identification: a probabilistic approach, AIAA Journal 36 (1998), $2137-2145$.

[15] J. Araujo, M. Mota, A. Mota and M. Maia, Structural damage identification: influence of model incompletes and errors, Composite Structures 62 (2003), 303-313.

[16] U. Lee, A reduced-domain method of structural damage identification: Application to a spectral element beam model, Shock and Vibration 10(5-6) (2003), 313-324.

[17] A. Teughles and G. De Roeck, Structural damage identification of the highway bridge Z24 by FE model updating, Journal of Sound and Vibration 278 (2004), 589-610.

[18] F. Garcés, C. Genatios, M. Lafuente and A. Mebarki, Dynamic Identification of Shear Wall Buildings: influence of mass models and nonparametric mass normalization on stiffness assessment, Proceedings of Eurodyn 2005 "European Conference on Dynamics- Structural Dynamics", Millpress Ed., Rotterdam , 2005, 1829-1833.

[19] J. Ricles and J. Kosmatka, Damage detection in elastic structures using vibratory residual forces and weighted sensitivity, AIAA Journal 30 (1992), 2310-2316. 
[20] P. Yuan, Z. Wu and X. Ma, Estimated mass and stiffness matrices of shear building from modal test, Earthquake Engineering and Structural Dynamics 27 (1998), 415-421.

[21] R. Ambrosini, J. Riera and R. Danesi, Analysis of structures subjected to random wind loading by simulation in the frequency domain, Probabilistic Engineering Mechanics 17 (2002), 233-239.

[22] SAP2000 SAP 2000. Analysis Reference Manual. Computers and Structures, Inc. Berkeley, California, USA. Version 8.0. 2002.

[23] H. Sohn and K. Law, Application of load-dependent Ritz vectors to Bayesian probabilistic damage detection, Probabilistic Engineering Mechanics 15 (2000), 139-153.

[24] MATLAB The Language of Technical Computing. The Math Works, Inc., 2000.

[25] P. García, C. Genatios, F. Garcés and M. Lafuente, Sadex: sistema computacional para la simulación e identificación de estructuras, Boletín Técnico del IMME 44 (2005), 21-32.

[26] A. Cunha and E. Caetano, From Input-Output to Output-Only Modal Identification of Civil Engineering Structures, Keynote Lecture at 1st International Operational Modal Analysis Conference. IOMAC (2005), Copenhagen, Denmark. 

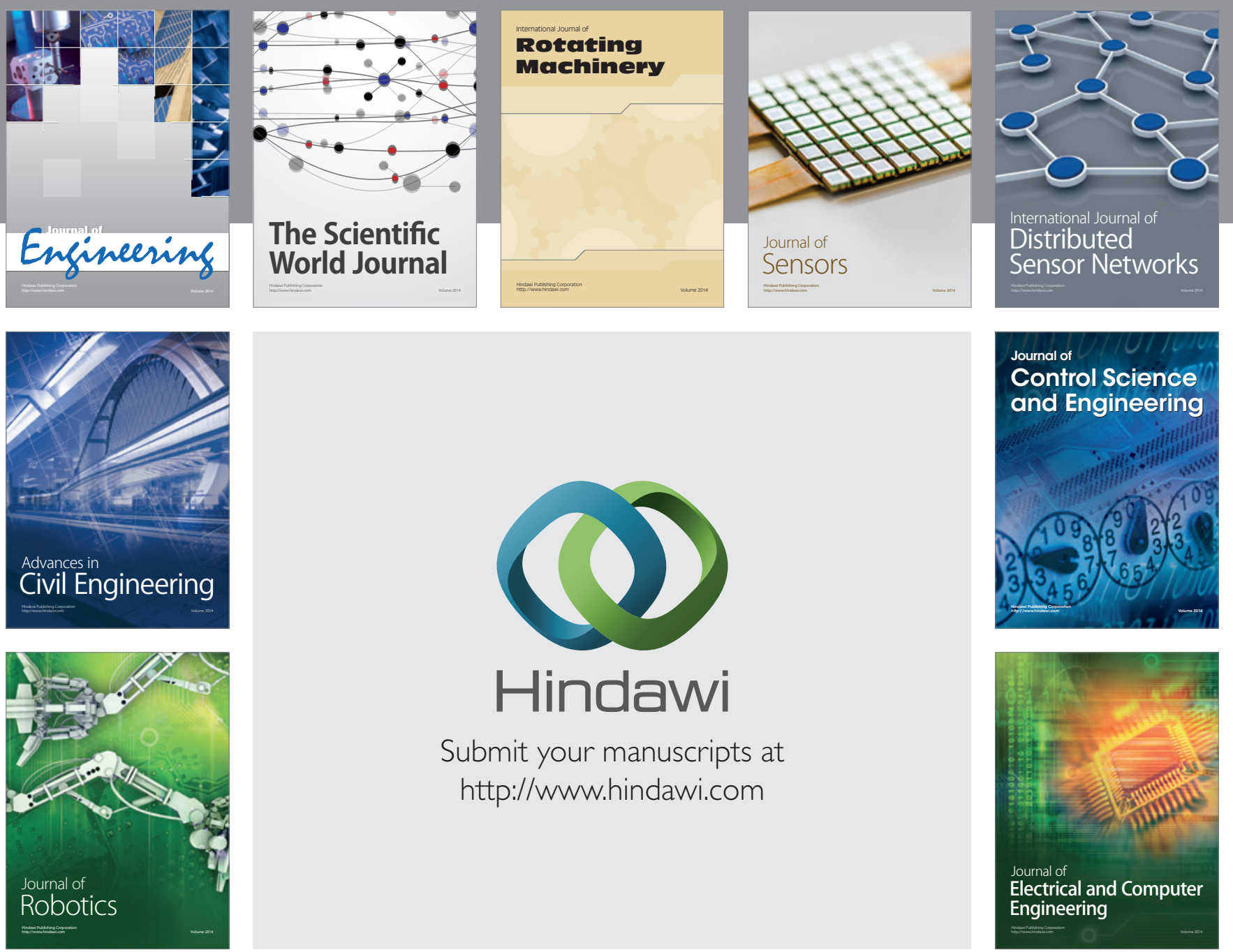

Submit your manuscripts at

http://www.hindawi.com
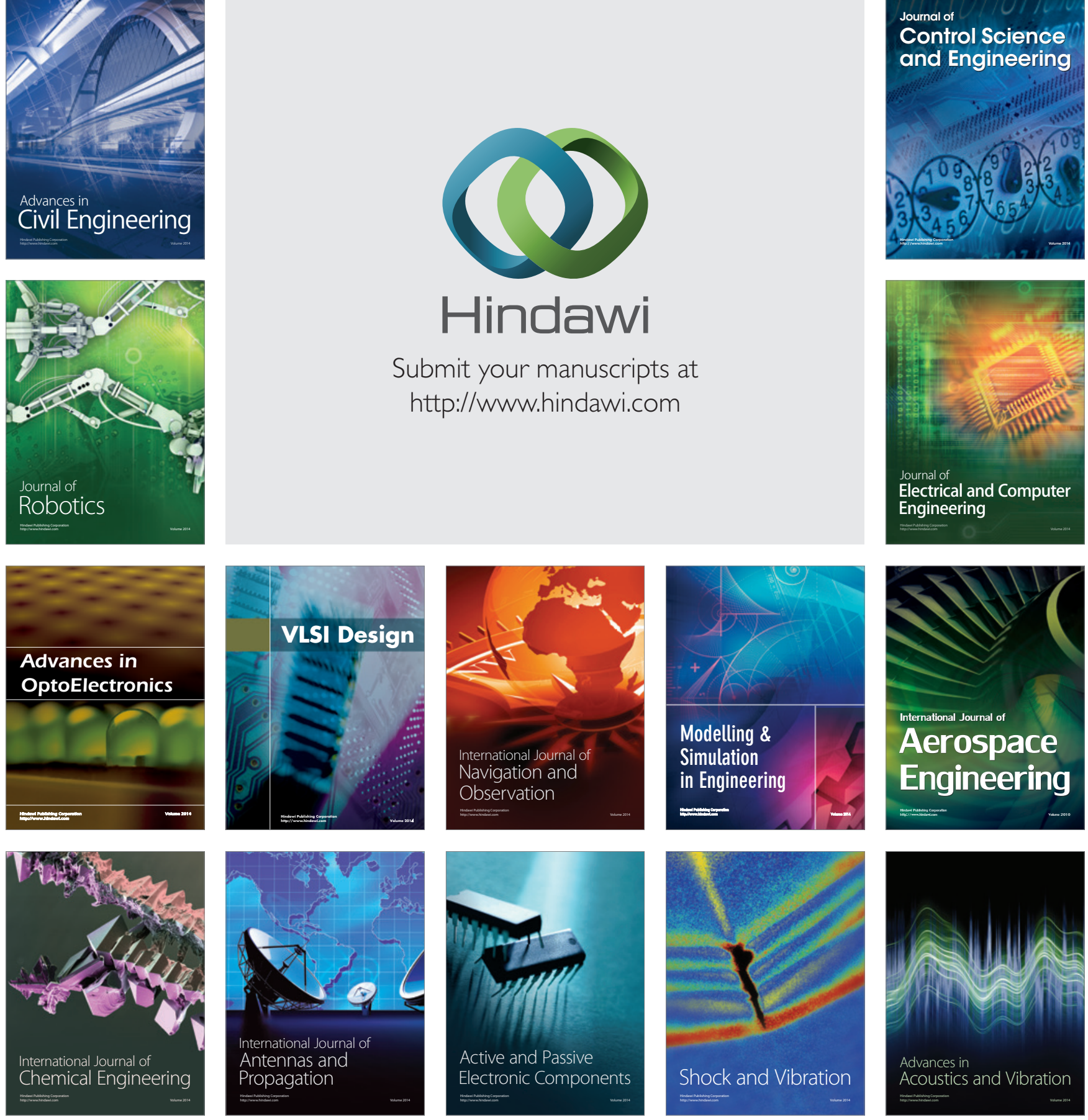